\title{
Learning Digital Control Design Made Easy through REAL-TIME EXPERIMENT SOLUTIONS
}

\author{
Yang Cao \\ School of Engineering \\ University of British Columbia, Okanagan Campus \\ Email: yang.cao@ubc.ca
}

\begin{abstract}
When teaching digital control course, it s found that students are often struggling with understanding the connection between the underlying mathematics for various control algorithms and their implementation. In particular, the effect of the control algorithm on system seems to be a mystery. Matlab simulation is able to help students better understanding the control system and building up confidence in the effectiveness of the controller. However, simulation alone is not able to get rid of questions such as "is it really going to work on real system?" or "how is it going to work in real-life?". This paper describes the integration of real-time experiment solutions into the digital control course offered in the School of Engineering at University of British Columbia Okanagan and gives detailed presentations on real-time implementation of digital control algorithms. In particular, implementation of $L Q G$ will be demonstrated. The impact on teaching and learning will also be discussed.
\end{abstract}

Keywords: Digital controller design, simplified implementation, motivated learning.

\section{Introduction}

Design of digital control systems are often based on abstract mathematical concepts and complex dynamic modeling. It is always desired by students to go beyond tedious mathematical equations and see the actual functioning the control algorithms. Simulations using Matlab/Simulink are able to help students better understand the control system and building up confidence in the effectiveness of the controller. However, the most critical needs for control engineers are to be able to integrate control algorithms and implement in practical applications [3]. Complete satisfaction comes from the smile when seeing real system turning or moving as commanded by the controller.

There are many commercially available educational control system products, among which Quanser SRV02 rotary workstation as shown in Fig. 1 is an ideal platform to teach control concepts. It includes the SRV02 plant, amplifier, data acquisition card, and control design software QuaRC [5]. The SRV02 plant provides three sensory information of the motor shaft. Potentiometer and Encoder give analog and digital position signal. A Tachometer gives shaft velocity. One can mount additional modules to the workstation, such as flexible link or flexible joint arm. Many researchers have used Quanser's SRV02 set-up [1, 6].

When installed, QuaRC is integrated with Matlab Simulink [6]. A set of Simulink library block can be used for building control system. One can simply drag and drop corresponding blocks from the library to Simulink diagram. Figure 2 shows the QuaRC library and Figure 3 illustrates a simple example of making use of the HIL Write block for moving the motor and the HIL Read block to display the current position of the motor shaft.

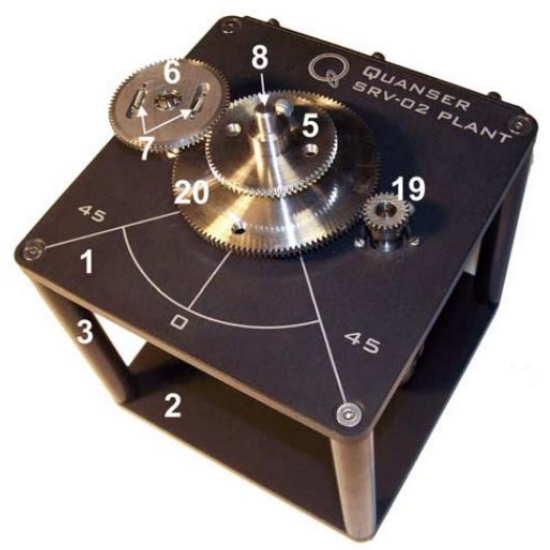

Fig. 1. SRV02 rotary workstation. 


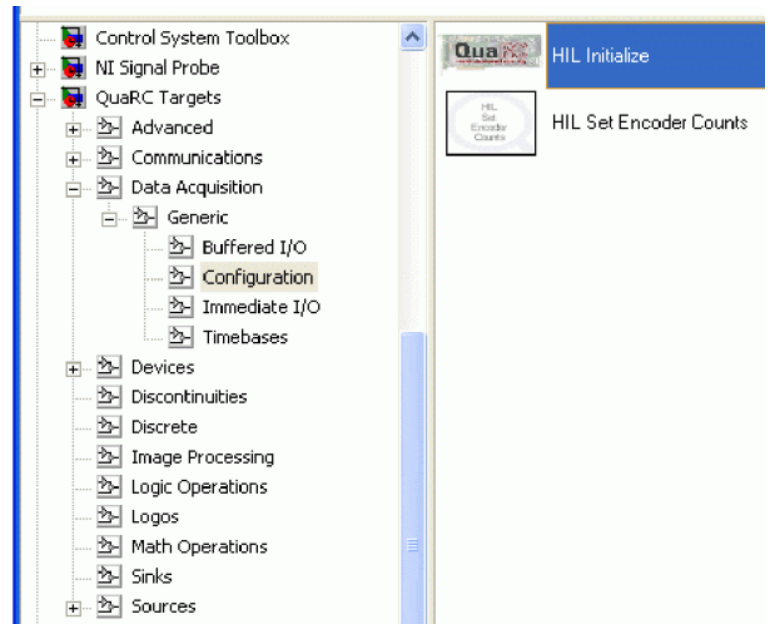

Fig. 2. QuaRC Configuration folder in the Simulink Library Browser window
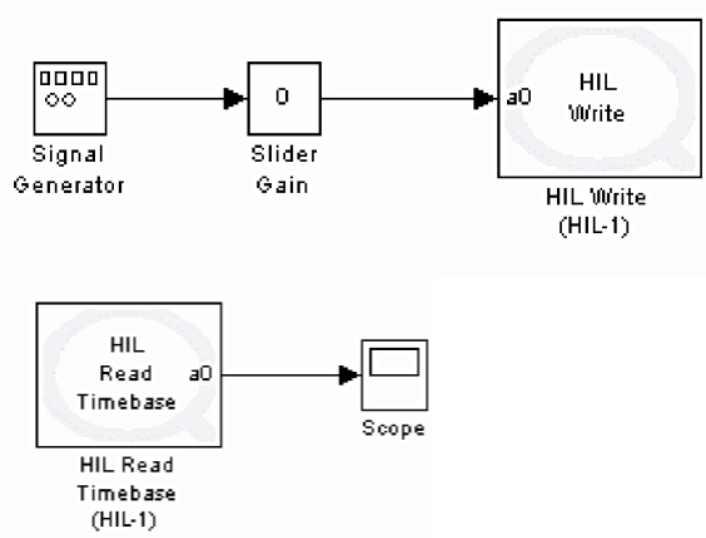

Fig. 3. Example of driving the motor and reading position information.

QuaRC provides a single PC solution for rapid control prototyping. It works with Matlab Real-Time workshop to generate real-time code directly from Simulinkdesigned controllers. In practice, one can replace the signal generator in Fig. 3 with designed controller. This hardware-in-the-loop (HIL) solution avoids the steep curve of learning programming language and significantly accelerates control design and implementation. It also helps students focus on the important aspects of the control design process and complete project-based assignments successfully.

This paper will describe the integration of the real-time experiment solutions into the digital control course offered in the School of Engineering at University of British Columbia Okanagan and gives detailed presentations on the frame work of how various digital control algorithms can be implemented in real-time.

\section{Structure of the Course}

"Digital Control" course (ENGR487) is a selective course offered to $4^{\text {th }}$ year mechanical and electrical engineering students in the School of Engineering at University of British Columbia Okanagan. The philosophy of emphasizing on real-time implementation of control system is reflected in the curriculum. Students are required to work in group of mixed mechanical and electrical engineering students to finish a term project on implementing a designed digital controller onto the Quanser Rotary experiment set-up. Students are can choose to implement various digital control schemes including discrete PID, pole placement, discrete Linear Quadratic Regulator (LQR), discrete Linear Quadratic Gaussian (LQG) and Recursive Least Square (RLS) for system identification.

\subsection{Pre-experiment work-Modeling and Simulation}

Students are required to model the SRV02 rotary high gear set-up and derive the transfer function between voltage input and position output. The transfer function between the position of load shaft and the input voltage can be found that

$$
\frac{\Theta_{l}(s)}{V_{m}(s)}=\frac{K}{s(\tau s+1)}
$$

where the $\Theta_{l}(s)$ is the Laplace transform of the load shaft position $\theta_{l}(t), V_{m}(s)$ is the Laplace transform of motor input voltage $v_{m}(t), K$ is the steady-state gain, $\tau$ is the time constant. It can be found that

$$
K=1.53 \frac{\mathrm{rad}}{\mathrm{sV}} \quad \tau=0.0253 \mathrm{~s}
$$

Based on the derived model, students do the following simulations using Matlab synchronized with the lectures.

(1) Obtain a $\mathrm{ZOH}$ equivalent discrete model for the plant with a sampling time $T_{s}=0.01$.

(2) Design a discrete PID controller and tune the gains to observe system response.

(3) Design a discrete optimal LQR based on the discrete model. Simulate using Matlab m-file.

(4) Design a discrete Kalman filter based on the discrete model. You can assume variance matrices for the process and sensor noise. Simulate using Matlab Simulink.

(5) Least square and Recursive least square system identification using $\mathrm{m}$-file.

The complexness of the controller design gradually increases. Starting from simple PID controller gives students confidence and a chance of getting familiar with Matlab. 


\subsection{Real-time Implementation}

Real-time implementation goes parallel with the simulation steps described in section 2.1. Once simulation runs satisfactorily, students are ready to implement on the SRV02 set-up. To facilitate the implementation, the following Simulink block representing the SRV02 model is provided (Fig. 4). Details of this masked block can be found in [6].
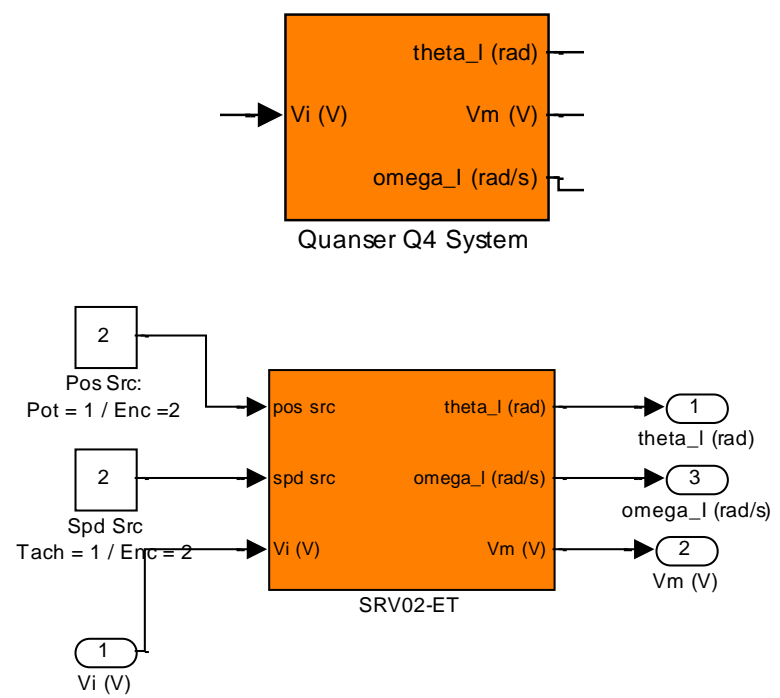

Fig. 4. Masked Simulink block for the SRV02 model.

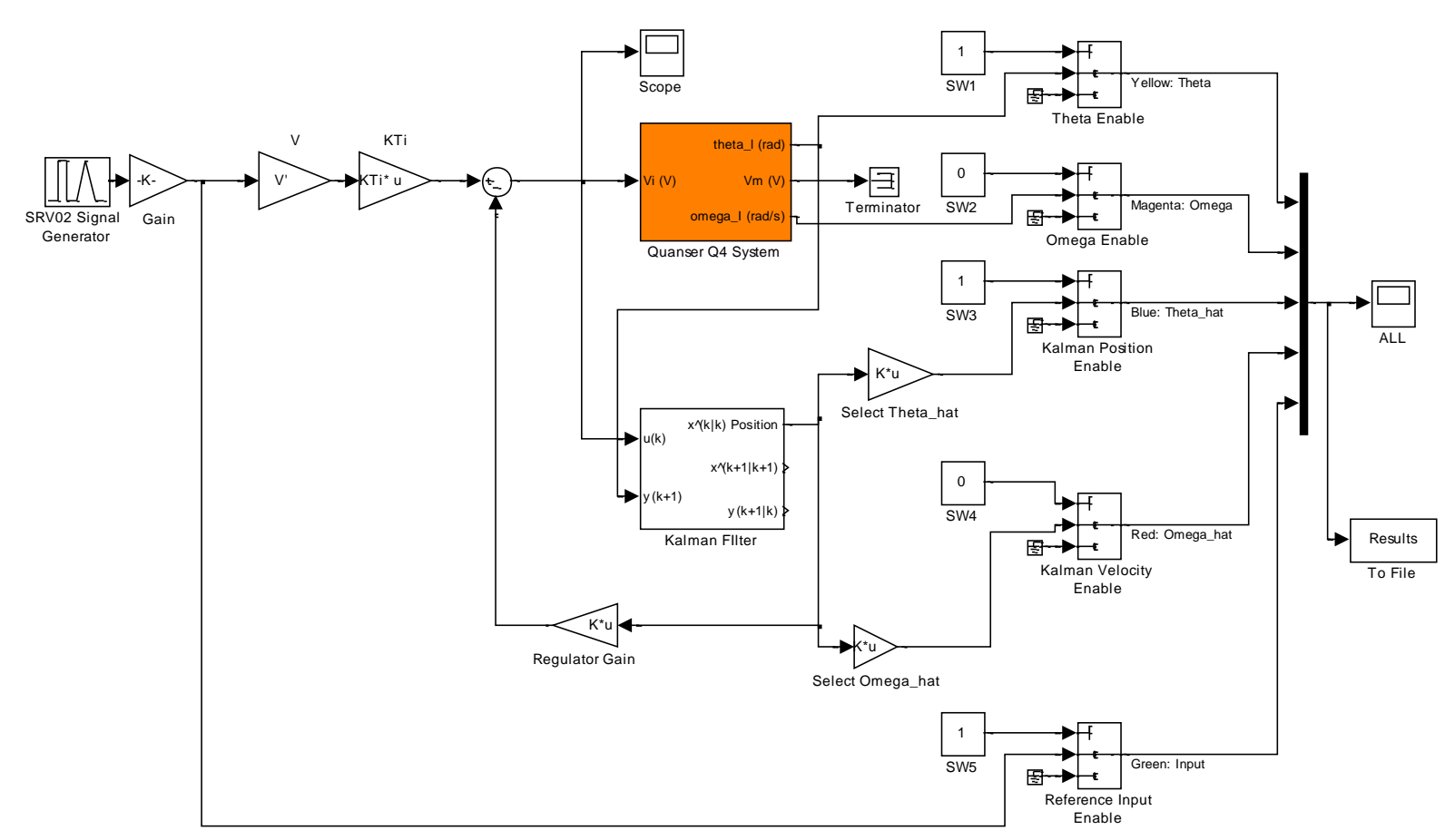

Fig. 5. Sample LQG controller implemented by students.
With the provided SRV02 Simulink block, students are able to focus on implementing their controllers knowing where to get the feedback of the plant and where to send control signal. The Simulink diagram shown in Figure 5 illustrates the implementation of discrete LQG controller by a group of students. LQG control provides optimal control (LQR) and optimal estimation (Kalman filter) of the system. Kalman filter is designed to estimate both position and velocity assuming white noise in the measurement from sensors. The LQR reference tracking control and Kalman filter algorithms [4] are simulated prior to implementation.

When the reference input is a sinusoidal signal, the response of the system is shown in Fig. 6 and Fig. 7. Simulation results verified the performance of the control algorithm and students can change gains to observe the change of response. 


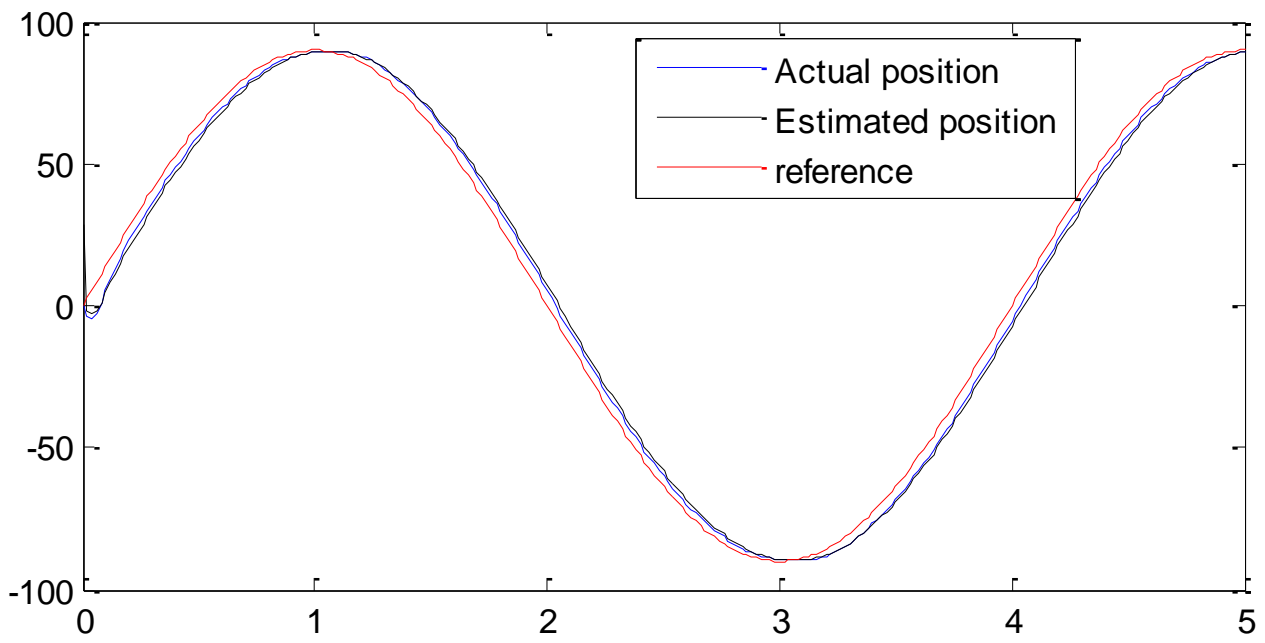

Fig. 6. System response (angular position) given sinusoidal input

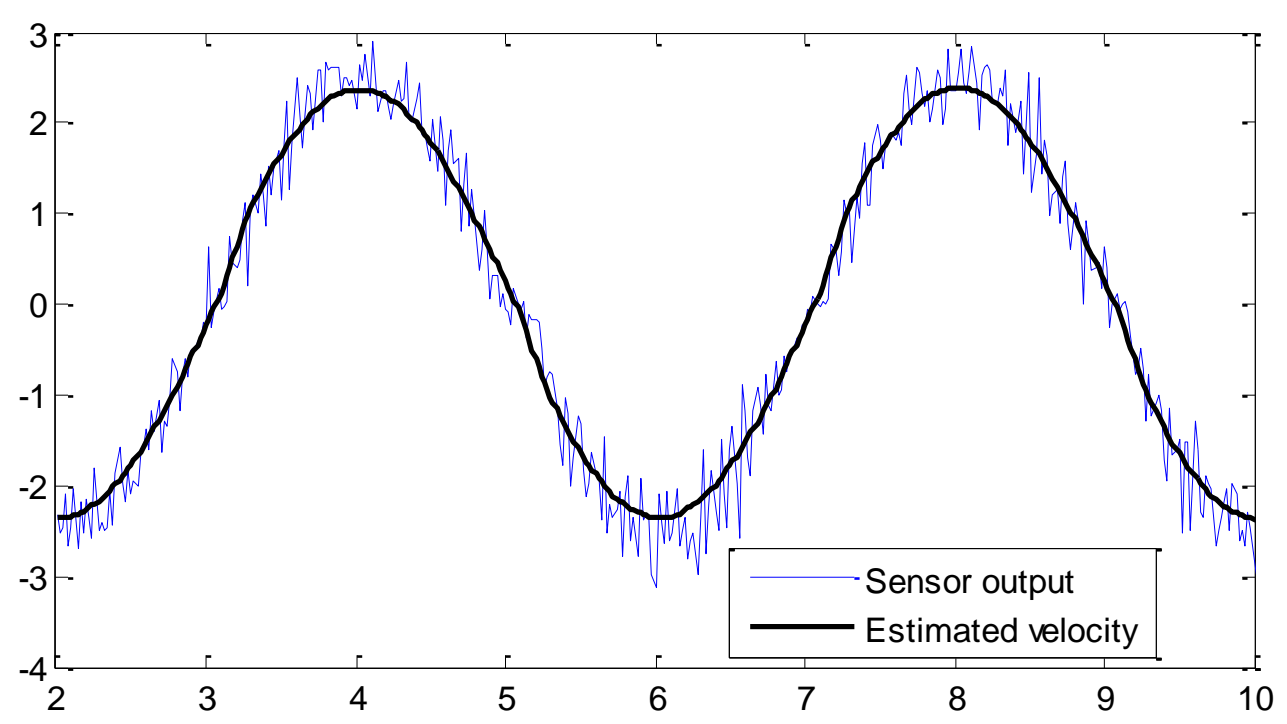

Fig. 7. Angular velocity from sensor and estimated one from Kalman filter.

\section{Observations, Lesson Learned and Future Improvement}

It is noticed that some students lack basic knowledge of Matlab/Simulink programming. To facilitate students learning Matlab and getting familiar with the project, assignments were designed to complement the purpose. From modeling to simulation of the experiment set-up, students gain experience in controller implementation. Real-time implementation is new to most of the students. Overall, students have shown strong interest in real-time experiment. Starting with discrete PID familiarizes them with the experiment set-up. Gradually increasing the difficulty of controller design meets different level of students learning.

The addition of the real-time implementation does add challenge to both teaching and learning. However, the success from simulation to real-time implementation gives students complete satisfaction and appreciation of the control algorithm. One of the most frequent phrases I hear when talking to students is "Amazing, that really works!" With the confidence and better understanding of LQR control, some groups of students even went beyond the project requirement and implemented swing- 
up and balancing controller for the rotary inverted pendulum system.

No formal surveys were conducted regarding students experience of learning digital control through experiment. However, due to the small class size, close monitoring and frequent communications with the students were maintained throughout the term. Students progresses were kept in pace with this phased project. No one is left behind with help from instructor; no matter it is programming, modeling, or algorithm implementation.

It is observed that students' frustrations mainly arise from tuning or debugging their Matlab program. Proper tuning and logical debugging are based on understanding of the control theories. In the end, the process of tuning and debugging helps student gain indepth understanding of theories.

Students are able to learn because they think they are having fun. For future improvement, classroom lecturing can be moved into the lab. Traditional lecture can be replaced with on-site demonstrations or practices.

\section{Conclusions}

Digital control is a challenge subject involving intensive mathematics, modeling, design and programming. Tradition method of teaching tends to cause frustration among students. Although Matlab simulation provides help in understanding the abstract theories, engineering students need to see it to believe it. Integrating real-time implementation into the current digital control course proves to be effective in motivating students. Real-time implementation provides students hands-on experience and proves to be a great aid for teaching and learning the principles of digital control. Through the experience of experiment, students were also prompted to notice the similarities and differences between mathematical simulation results and response from actual system. The outcome of this approach is well accepted and highly rated by students.

\section{References}

[1] Svante Gunnarsson, "Iterative learning control of a flexible robot arm using accelerometers", in Proc. of the 2004 IEEE International Conference on Control Applications, Vol.2, pp. 1012-1016, 2004.

[2] Cosmin Ionete, "LQG/LTR Controller Design for Flexible Link Quanser Real-time Experiment", in Proc. of Int. Symp. SINTES11, Craiova, Romania, 2003, vol. 1, pp. 4954.

[3] Chyi-Shyong Lee, Juing-Huei Su, Yu-Li Wang, Chia-Ruei Lee, and Jia-Shing Fu, "Learning digital control systems with a low-cost educational platform", in Proceedings of 2005 CACS Automatic Control Conference, Tainan, Nov $18-19,2005$

[4] Katsuhiko Ogata, Discrete-time Control System, PrenticeHall Inc., New Jersey, 1995.

[5] Quanser SRV02 User Manual.

[6] Quanser QuaRC Installation Manual 